\title{
Appropriate Use of CT Perfusion following Aneurysmal Subarachnoid Hemorrhage: A Bayesian Analysis Approach
}

R.P. Killeen, A. Gupta, H. Delaney, C.E. Johnson, A.J. Tsiouris, J. Comunale, M.E. Fink, H.S. Mangat, A.Z. Segal, A.I. Mushlin, and P.C. Sanelli

\begin{abstract}
BACKGROUND AND PURPOSE: In recent years CTP has been used as a complementary diagnostic tool in the evaluation of delayed cerebral ischemia and vasospasm. Our aim was to determine the test characteristics of CTP for detecting delayed cerebral ischemia and vasospasm in $\mathrm{SAH}$, and then to apply Bayesian analysis to identify subgroups for its appropriate use.
\end{abstract}

MATERIALS AND METHODS: Our retrospective cohort comprised consecutive patients with SAH and CTP performed between days 6 and 8 following aneurysm rupture. Delayed cerebral ischemia was determined according to primary outcome measures of infarction and/or permanent neurologic deficits. Vasospasm was determined by using DSA. The test characteristics of CTP and its $95 \%$ Cls were calculated. Graphs of conditional probabilities were constructed by using Bayesian techniques. Local treatment thresholds (posttest probability of delayed cerebral ischemia needed to initiate induced hypertension, hypervolemia, and hemodilution or intra-arterial therapy) were determined via a survey of 6 independent neurologists.

RESULTS: Ninety-seven patients with SAH were included in the study; 39\% (38/97) developed delayed cerebral ischemia. Qualitative CTP deficits were seen in 49\% (48/97), occurring in 84\% (32/38) with delayed cerebral ischemia and 27\% (16/59) without. The sensitivity, specificity, and positive and negative predictive values (95\% CI) for CTP were 0.84 (0.73-0.96), 0.73 (0.62-0.84), 0.67 (0.51-0.79), and 0.88 (0.74-0.94), respectively. A subgroup of 57 patients underwent DSA; 63\% (36/57) developed vasospasm. Qualitative CTP deficits were seen in 70\% (40/57), occurring in $97 \%$ (35/36) with vasospasm and $23 \%(5 / 21)$ without. The sensitivity, specificity, and positive and negative predictive values $(95 \% \mathrm{Cl}$ ) for CTP were 0.97 (0.92-1.0), 0.76 (0.58-0.94), $0.88(0.72-0.95)$, and $0.94(0.69-0.99)$, respectively. Treatment thresholds were determined as $30 \%$ for induced hypertension, hypervolemia, and hemodilution and $70 \%$ for intra-arterial therapy.

CONCLUSIONS: Positive CTP findings identify patients who should be carefully considered for induced hypertension, hypervolemia, and hemodilution and/or intra-arterial therapy while negative CTP findings are useful in guiding a no-treatment decision.

ABBREVIATIONS: aSAH = aneurysmal subarachnoid hemorrhage; $\mathrm{DCl}=$ delayed cerebral ischemia; $\mathrm{GCP}=$ graph of conditional probabilities; $\mathrm{HHH}=$ induced hypertension, hypervolemia, and hemodilution

A neurysmal subarachnoid hemorrhage (aSAH) is a devastating condition, with complications of vasospasm and delayed cerebral ischemia (DCI) resulting in significant morbidity and

Received February 15, 2013; accepted after revision July 1.

From the Departments of Radiology (R.P.K., A.G., H.D., C.E.J., A.J.T., J.C., P.C.S.), Neurology (M.E.F., H.S.M., A.Z.S.), and Public Health (A.I.M., P.C.S.), Weill Cornell Medical College, NewYork-Presbyterian Hospital, New York, New York.

This work was supported by grant 5K23NS058387-04 from the National Institute of Neurological Disorders and Stroke, a component of the National Institutes of Health.

Paper previously presented in part at: Annual Meeting of the American Society of Neuroradiology and the Foundation of the ASNR Symposium, New York; April 21-26, 2012.

The views herein are solely those of the authors and do not necessarily represent the official view of the National Institute of Neurological Disorders and Stroke or the National Institutes of Health. mortality. The pathophysiology of vasospasm and DCI is complex and poorly understood, leading to delayed diagnosis and treatment. ${ }^{1}$ Currently, both clinical and imaging findings are used to detect vasospasm and DCI. The clinical findings of new symptoms not attributed to other known causes coupled with imaging evidence of vasospasm may prompt treatment to prevent DCI and subsequent infarction. The diagnosis remains challenging because many patients are critically ill, precluding thorough clinical assessment, ${ }^{2}$ and discrepant findings between clinical and imaging examinations lead to indeterminate diagnoses. Prior studies have reported that almost half of patients with severe vasospasm

Please address correspondence to Pina C. Sanelli, MD, MPH, Department of Radiology, Weill Cornell Medical College, New York-Presbyterian Hospital, 525 East 68th St, Starr 8A, Box 141, New York, NY 10065; e-mail: pcs9001@med.cornell.edu

- Indicates open access to non-subscribers at www.ajnr.org

http://dx.doi.org/10.3174/ajnr.A3767 
do not have DCI. ${ }^{3}$ Additionally, DCI is not always attributed to vasospasm and may occur in the absence of arterial narrowing, indicating that the relationship between vasospasm and DCI is not completely understood. Thus, it is important to consider vasospasm and DCI as 2 related but somewhat different clinical entities. DCI has been recently defined by expert consensus by using the primary outcome measures of functional disability and/or cerebral infarction not attributed to other causes, ${ }^{1}$ and "vasospasm" has been defined as arterial narrowing seen on imaging studies.

In recent years, CTP has been added as a complementary diagnostic tool for determining vasospasm and DCI. Several reports describe its high sensitivity and specificity to detect perfusion abnormalities thought to occur in both vasospasm and DCI. ${ }^{3-8} \mathrm{CTP}$ has several other advantages in this critically ill population, including its widespread access, acquisition speed, and few patient contraindications. However, CTP also has disadvantages, with the main concern being increased radiation exposure relative to noncontrast CT of the head, due to its cine scanning technique. ${ }^{9} \mathrm{Bi}-$ ologic effects from radiation exposure, including temporary epilation and erythema, have been described in patients with aSAH and acute stroke who have undergone CTP. ${ }^{10,11}$ As a result, the FDA issued a notification to health care providers to promote radiation safety and appropriate use of CT imaging. ${ }^{11}$ Currently, there are no guidelines for the appropriate use of CTP in aSAH, which may potentially result in overuse of CTP with unnecessary radiation exposure, contrast reactions, and increased cost. There is also potential for underuse, in which the diagnosis of vasospasm and DCI may remain elusive and patients will not receive optimal and timely treatment. Thus, it is important to identify patients who will benefit most from CTP to outweigh its associated risks.

Our hypothesis is that application of Bayesian analysis ${ }^{12-15} \mathrm{can}$ guide appropriate use of CTP in aSAH by identifying subgroups in which CTP results affect treatment decisions. Bayesian analysis uses probability theory to calculate the posttest probability of vasospasm or DCI following a positive or negative CTP finding. The purpose of this study was to determine the test characteristics of qualitative CTP for identifying 2 separate but related entities in aSAH, angiographic vasospasm and DCI, and then to apply Bayesian analysis to identify subgroups for its appropriate use.

\section{MATERIALS AND METHODS \\ Study Design}

We performed a retrospective study of consecutive patients with aSAH enrolled in an institutional review board-approved clinical accuracy trial from December 2004 to December 2008. Inclusion criteria were adult patients ( 18 years and older) with documented aSAH at admission determined by initial noncontrast head CT, CSF analysis, CTA, and/or DSA. Exclusion criteria were CTP examinations with severe motion degradation and CTP examinations performed after infarction or treatment of vasospasm had occurred. All patients underwent surgical clipping and/or endovascular coiling for aneurysm repair and were monitored in the neurointensive care unit, as per the usual standard-of-care procedures.

Chart review was performed for the clinical and demographic characteristics of the study population. During hospitalization, patients were assessed for symptoms of vasospasm and DCI, defined as delayed onset of neurologic deterioration, which was not explained by other causes, such as aneurysm rebleeding, intracranial hemorrhage, hydrocephalus, infection, metabolic disturbance, seizure, and so forth. Neurologic deterioration may manifest as alterations in consciousness, worsening on the Glasgow Coma Scale, or new neurologic deficits (aphasia, paresis, and so forth). In patients with suspected vasospasm or DCI, based on neurologic deterioration and transcranial Doppler sonography, DSA with the potential for endovascular treatment was performed. Management decisions were based on all clinical and imaging data, except CTP.

\section{CTP Protocol and Data Processing}

CTP was performed during the typical time of vasospasm and DCI, between days 6 and 8 in asymptomatic patients (patients without neurologic deterioration) and on the same day that neurologic deterioration occurred in symptomatic patients. Of note, CTP examinations were performed at the diagnostic stage, before treatment for vasospasm or DCI and before patients developed infarction. There is a standard scanning protocol for CTP at our institution by using LightSpeed or Pro 16 scanners (GE Healthcare, Milwaukee, Wisconsin) with a cine $4 \mathrm{i}$ scanning mode and a 45 -second acquisition at 1 rotation per second by using $80 \mathrm{kV}$ (peak) and $190 \mathrm{~mA}$. To minimize radiation exposure to the lenses, we used a scanning volume of $2.0 \mathrm{~cm}$ with its inferior extent selected above the orbits and at the level of the basal ganglia. Approximately $45 \mathrm{~mL}$ of nonionic iodinated contrast was administered intravenously at $5 \mathrm{~mL} / \mathrm{s}$ by using a power injector with a 5-second delay.

Postprocessing of the acquired images into MTT, CBF, and CBV maps was performed on an Advantage Workstation (GE Healthcare) by using CT Perfusion software, Version 3.0 (GE Healthcare). The postprocessing technique was standardized for all patients according to recommended guidelines, with the arterial input function as the A2 segment of the anterior cerebral artery and the venous function as the superior sagittal sinus. ${ }^{16}$

The perfusion maps were qualitatively evaluated by 2 neuroradiologists (with 10 and 7 years' experience, respectively) to determine the presence of perfusion deficits, defined as areas of decreased CBF and/or prolonged MTT. Focal perfusion abnormalities due to the primary hemorrhagic event and/or surgical intervention were not considered as perfusion deficits from vasospasm or DCI. After reviewing the images independently, consensus judgment was determined. CTP examinations were analyzed blinded to all clinical and imaging data to limit test-review bias.

\section{Reference Standard for Vasospasm and DCI}

Vasospasm and DCI were assessed by 2 separate reference standards. The diagnosis of vasospasm was based on angiographic criteria by using DSA to determine arterial luminal narrowing compared with the healthy parent vessel and with DSA performed on the initial presentation. Arterial narrowing of $>50 \%$ compared with the parent vessel was classified as vasospasm. DSA interpretations were performed by 2 observers, an interventional neuroradiologist who performed the examination (with either 10 or 25 years' experience) and a neuroradiologist blinded to all clin- 
Table 1: Clinical and demographic characteristics of the study population and subgroups

\begin{tabular}{|c|c|c|c|c|c|c|}
\hline & \multicolumn{3}{|c|}{ Study Population } & \multicolumn{3}{|c|}{ Subgroup } \\
\hline & \multicolumn{3}{|c|}{$(n=97)$} & \multicolumn{3}{|c|}{ (DSA as Reference Standard) $(n=57)$} \\
\hline & All $(n=97)$ & $\mathrm{DCl}(n=38)$ & No DCl $(n=59)$ & All $(n=57)$ & Vasospasm $(n=36)$ & No vasospasm $(n=21)$ \\
\hline Age (yr) (median) & 49 & 54 & 48 & 51 & 48 & 55 \\
\hline (Range) & $28-80$ & $34-78$ & $28-80$ & $28-80$ & $30-78$ & $28-80$ \\
\hline \multicolumn{7}{|l|}{ Sex (\%) (No.) } \\
\hline Male & $27(26 / 97)$ & $32(12 / 38)$ & $24(14 / 59)$ & $25(14 / 57)$ & $25(9 / 36)$ & $24(5 / 21)$ \\
\hline Female & 73 (71/97) & $68(26 / 38)$ & $76(45 / 59)$ & $75(43 / 57)$ & $75(27 / 36)$ & $76(16 / 21)$ \\
\hline \multicolumn{7}{|l|}{ Aneurysm location (\%) (No.) } \\
\hline Anterior & $92(89 / 97)$ & $92(35 / 38)$ & $93(55 / 59)$ & $95(54 / 57)$ & $92(33 / 36)$ & $100(21 / 21)$ \\
\hline Posterior & $8(8 / 97)$ & $8(3 / 38)$ & $7(4 / 59)$ & $5(3 / 57)$ & $8(3 / 36)$ & $0(0 / 21)$ \\
\hline \multicolumn{7}{|l|}{ Treatment type (\%) (No.) } \\
\hline Surgical clipping & $55(53 / 97)$ & $63(24 / 38)$ & $49(29 / 59)$ & $65(37 / 57)$ & $56(20 / 36)$ & $81(17 / 21)$ \\
\hline Coil embolization & $45(44 / 97)$ & $37(14 / 38)$ & $51(30 / 59)$ & $35(20 / 57)$ & $44(16 / 36)$ & $19(4 / 21)$ \\
\hline \multicolumn{7}{|c|}{ Hunt and Hess grade (\%) (No.) } \\
\hline Low (grades 1 and 2) & $53(51 / 97)$ & $37(14 / 38)$ & $61(36 / 59)$ & $46(26 / 57)$ & $36(13 / 36)$ & $62(13 / 21)$ \\
\hline High (grades 3, 4 and 5) & $47(46 / 97)$ & $63(24 / 38)$ & $39(23 / 59)$ & $54(31 / 57)$ & $64(23 / 36)$ & $38(8 / 21)$ \\
\hline
\end{tabular}

ical and imaging data (with 22 years' experience). For disagreements, a third neuroradiologist (with 10 years' experience) independently reviewed the DSA in a blinded fashion to determine consensus.

The reference standard for DCI was based on the expert consensus opinion ${ }^{1}$ by using the following outcome measures: 1) cerebral infarction demonstrated on CT or MR imaging within 6 weeks after aSAH, which was not present on imaging up to 48 hours after aneurysm occlusion and was not attributable to other causes such as surgical clipping, endovascular treatment, ventricular catheter placement, or intraparenchymal hematoma (this criterion for cerebral infarction has been used to effectively exclude primary brain damage from aSAH and/or surgical interventions ${ }^{17,18}$ ); and/or 2) permanent neurologic deficit on clinical examination, distinct from the deficit at baseline produced by the aneurysm rupture or surgical intervention and not attributable to other causes. Thorough chart review was performed and consensus was determined by expert neurologists (with 14 and 4 years' experience) and a neuroradiologist (with 10 years' experience) to classify patients according to these criteria.

\section{Statistical Analysis}

The sensitivity, specificity, and likelihood ratios were calculated for qualitative CTP deficits in determining vasospasm and DCI, separately. ${ }^{19}$ The $95 \%$ confidence interval was calculated as the measure of variance.

The entire spectrum of posttest probabilities was calculated for positive and negative CTP findings by multiplying the pretest odds and the positive and negative likelihood ratios, respectively, which are represented as a graph of conditional probability (GCP). Construction of the GCPs was performed by using an available data analysis Web-based spreadsheet (www.ebr.ie). ${ }^{12,20}$ The posttest probabilities are represented on the $y$-axis, and pretest probabilities, on the $\mathrm{x}$-axis. To illustrate the usefulness of CTP in the Bayesian analysis, we based selection of the pretest probabilities for vasospasm and DCI on commonly used clinical classification schemes in the literature, such as the Hunt and Hess scale, Fisher grade, modified Fisher scale, and Glasgow Coma Scale. ${ }^{21}$ Given the variability in pretest probabilities of DCI for each of these classification schemes, Bayesian analysis was applied to each classification scheme separately to determine posttest probabilities after CTP testing to demonstrate whether differences in CTP use existed among these prediction tools.

Locally observed treatment thresholds for initiating medical management with induced hypertension, hypervolemia, and hemodilution $(\mathrm{HHH})$ and intra-arterial therapy with vasodilatory agents and/or angioplasty were evaluated by an independent survey of 6 neurologists (range, 1-29 years' experience) at our institution, to demonstrate the role of CTP in decision-making. Each neurologist described the minimum posttest probability for DCI needed to initiate $\mathrm{HHH}$ or intra-arterial therapy by considering the benefits and risks of treatment. Review of survey results and median treatment thresholds were determined by an independent group of 2 other authors not involved in the survey.

\section{RESULTS}

\section{Study Population Characteristics}

Ninety-seven patients were included in the statistical analysis from the 104 patients enrolled in the prospective study. Seven patients were excluded for the following reasons: CTP examinations were not performed before treatment for vasospasm $(n=4)$, CTP acquired data were not retrievable from the archives for postprocessing $(n=2)$, and postprocessing could not be performed due to severe motion degradation $(n=1)$. The median age (range) was 49 years (28-80 years), and 73\% (71/97) were women. Ninety-two percent (89/97) of the aneurysms were located in the anterior circulation. The treatment for aneurysm repair in this study population was $55 \%$ (53/97) by surgical clipping and $45 \%$ (44/97) via endovascular coiling procedures. The clinical Hunt and Hess scale grades on presentation were 47\% (46/97) high grades 3, 4, and 5 and 53\% (51/97) low grades 1 and 2. Table 1) presents the demographic and clinical characteristics of the study population according to DCI and vasospasm groups.

According to the reference standard, DCI was diagnosed in $39 \%(38 / 97)$ of patients. Thirty-two percent (12/38) of patients who developed DCI were initially asymptomatic. For the vasospasm analysis, 57 patients were available who also had DSA performed to determine the reference standard. In this subgroup, vasospasm was diagnosed in 63\% (36/57) of these patients. 


\section{Qualitative CTP Analysis}

Day 7 was the median time when CTP was performed (range, 2-17 days). Qualitative CTP deficits were seen in 49\% (48/97) of the study population, occurring in $84 \%(32 / 38)$ of patients with DCI and 27\% (16/59) without DCI. The sensitivity, specificity, and positive and negative predictive values (95\% CI) of CTP for determining DCI were 0.84 (0.73-0.96), 0.73 (0.62-0.84), 0.67 (0.51-0.79), and $0.88(0.74-0.94)$, respectively. The positive and negative likelihood ratios (95\% CI) were $3.1(2.0-4.8)$ and 0.2 (0.10-0.46), respectively.

In the subgroup of patients who had DSA performed, qualitative CTP deficits were seen in 70\% (40/57), occurring in $97 \%$ $(35 / 36)$ of patients with vasospasm and $23 \%$ (5/21) of patients without vasospasm. The sensitivity, specificity, and positive and negative predictive values $(95 \% \mathrm{CI})$ of CTP for detecting vasospasm were 0.97 (0.92-1.0), 0.76 (0.58-0.94), 0.88 (0.72-0.95), and $0.94(0.69-0.99)$, respectively. The positive and negative likelihood (95\% CI) ratios were $4.1(1.9-8.8)$ and 0.04 (0.005-0.25), respectively. The median time between CTP and DSA was 1 day (range, 1-3 days).

\section{Bayesian Analysis}

The GCPs of CTP for determining DCI and vasospasm are shown in Fig $1 A,-B$, respectively. The GCP represents the spectrum of posttest probabilities for a positive or negative CTP test result over a range of pretest probabilities. Table 2 displays the pretest probabilities of DCI based on the classification schemes commonly used in clinical practice. To illustrate the effect of CTP, we calculated the posttest probabilities of DCI for each classification scheme when CTP yielded a positive or negative result.

The median treatment thresholds at our institution for $\mathrm{HHH}$ and intra-arterial therapy were $30 \%$ and $70 \%$, respectively, indicating that a patient with a posttest probability of $>30 \%$ following CTP should be considered for treatment with $\mathrm{HHH}$ and of $>70 \%$, for intra-arterial therapy. These treatment thresholds, which could vary by local practice pattern, are strictly used for illustrative purposes in this study to demonstrate the utility of CTP by using Bayesian analysis and have been incorporated in the GCPs (Fig 1). Overall, a positive CTP finding will increase the posttest probability of DCI above the $\mathrm{HHH}$ treatment threshold in any patient with a pretest probability of $\geq 12 \%$. On the other hand, a negative CTP finding will decrease the posttest probability below the $\mathrm{HHH}$ treatment threshold in any patient with a pretest probability of $<67 \%$. A positive CTP finding will increase the posttest probability of vasospasm above the $\mathrm{HHH}$ treatment threshold in any patient with a pretest probability of $\geq 10 \%$, and a negative CTP finding will decrease the posttest probability below the treatment threshold in any patient with a pretest probability of $<92 \%$.
Table 2: Pretest and posttest probabilities of DCI based on clinical classification schemes ${ }^{\mathrm{a}}$

\begin{tabular}{|c|c|c|c|}
\hline \multirow[b]{2}{*}{ Clinical Classification Schemes } & \multirow[b]{2}{*}{$\begin{array}{l}\text { Pretest Probability } \\
\qquad(95 \% \mathrm{Cl})\end{array}$} & \multicolumn{2}{|c|}{ Posttest Probability } \\
\hline & & $\begin{array}{l}\text { CTP-Positive } \\
\text { (95\% CI) }\end{array}$ & $\begin{array}{c}\text { CTP-Negative } \\
(95 \% \mathrm{Cl})\end{array}$ \\
\hline Hunt and Hess grades $1-2^{21}$ & $0.35(0.25-0.45)$ & $0.62(0.52-0.72)$ & $0.10(0.04-0.16)$ \\
\hline Hunt and Hess grades $3-5^{21}$ & $0.61(0.53-0.70)$ & $0.82(0.74-0.90)$ & $0.25(0.16-0.34)$ \\
\hline Glasgow Coma Scale $\geq 9^{21}$ & $0.45(0.38-0.53)$ & $0.72(0.63-0.81)$ & $0.15(0.08-0.22)$ \\
\hline Glasgow Coma Scale $<9^{21}$ & $0.71(0.57-0.86)$ & $0.88(0.82-0.95)$ & $0.35(0.26-0.45)$ \\
\hline Modified Fisher grade $1^{26}$ & $0.13(0.0-0.29)$ & $0.31(0.22-0.40)$ & $0.03(0.0-0.06)$ \\
\hline Modified Fisher grade $2,3^{26}$ & $0.20(0.11-0.28)$ & $0.44(0.34-0.54)$ & $0.05(0.01-0.09)$ \\
\hline Modified Fisher grade $4^{26}$ & $0.36(0.24-0.47)$ & $0.66(0.57-0.75)$ & $0.11(0.05-0.17)$ \\
\hline
\end{tabular}

a The pretest probabilities are based on literature review and the posttest probabilities are calculated from the CTP test characteristics derived from this study.

\section{DISCUSSION}

The application of CTP for detecting vasospasm and DCI has been increasing in patients with aSAH partly due to its high sensitivity and specificity reported in the literature. ${ }^{6,8,22-24}$ Our sensitivity (95\% $\mathrm{CI})$ of $0.84(0.73-0.96)$ and specificity (95\% CI) of $0.73(0.62-0.84)$ for CTP detecting DCI are similar to those reported in the literature. ${ }^{3,4}$ Additionally, we report a sensitivity $(95 \% \mathrm{CI})$ of $0.97(0.92-$ 1.0 ) and a specificity (95\% CI) of 0.76
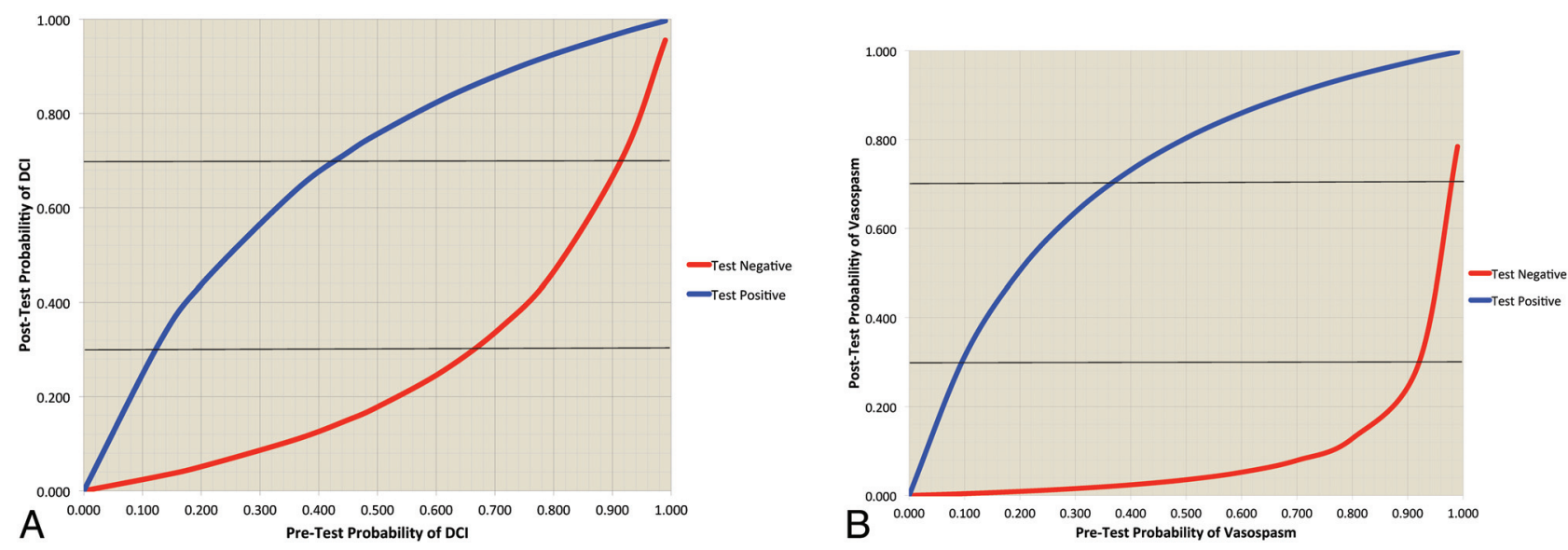

FIG 1. A, Graph of conditional probabilities for CTP determining DCI in aSAH. The blue curve represents the spectrum of posttest probabilities for a positive CTP finding. The red curve represents the spectrum of posttest probabilities for a negative CTP finding. The horizontal black lines represent the treatment threshold posttest probabilities of $30 \%$ and $70 \%$ for $\mathrm{HHH}$ and intra-arterial therapy, respectively. B, Graph of conditional probabilities for CTP determining vasospasm in aSAH. The blue curve represents the spectrum of posttest probabilities for a positive CTP finding. The red curve represents the spectrum of posttest probabilities for a negative CTP finding. The horizontal black lines represent the posttest probability treatment thresholds of $30 \%$ and $70 \%$, for $\mathrm{HHH}$ and intra-arterial therapy, respectively. 

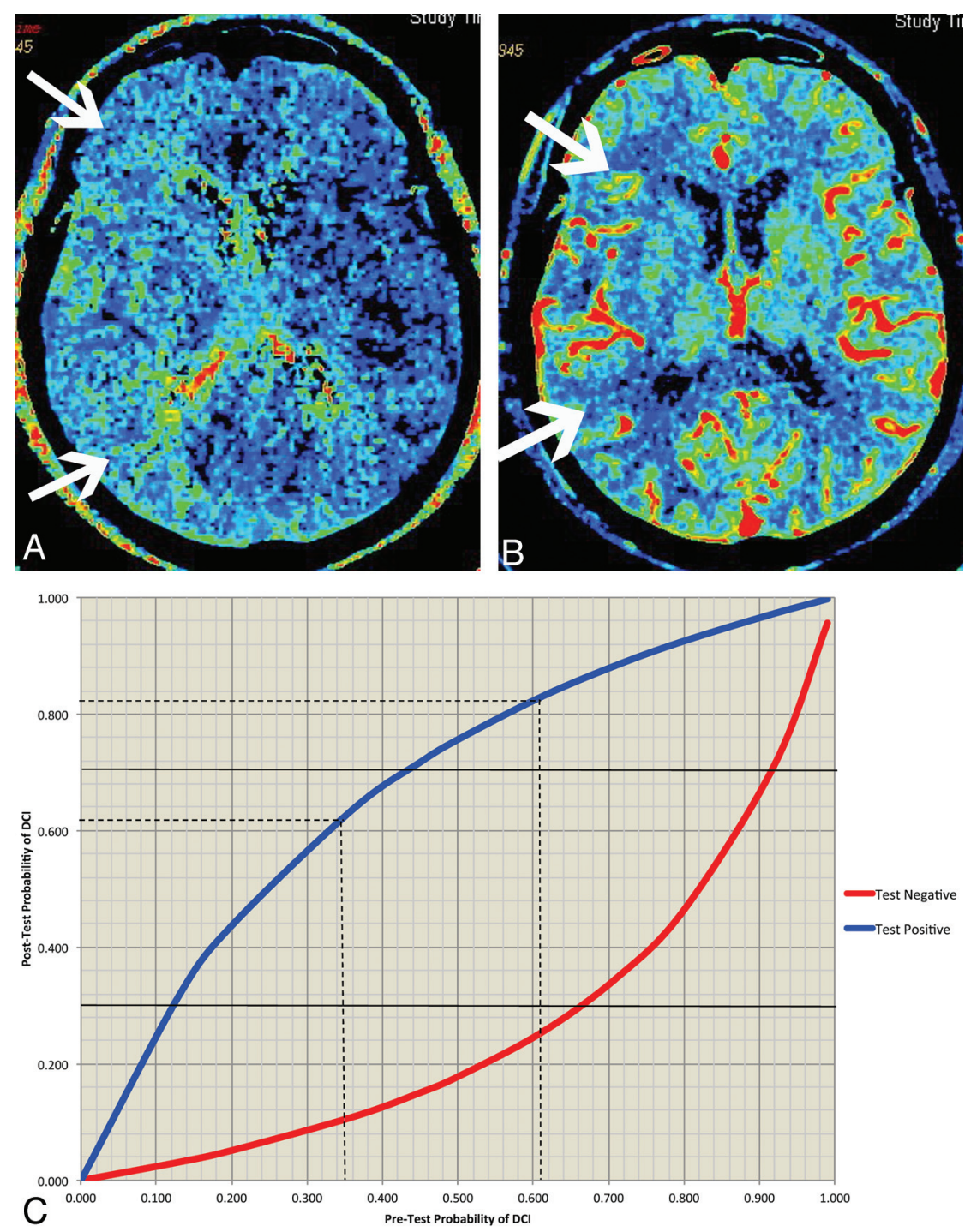

FIG 2. A 65-year-old woman with a CTP examination 6 days following aSAH. A, The MTT map demonstrates diffuse prolongation of MTT in the vascular territory of the right middle cerebral artery (arrows). B, The CBF map from the same level demonstrates a reduction in CBF in a similar distribution. C, The GCP for DCl is customized for this patient on the basis of the Hunt and Hess scale scores, as a sample clinical predictor. The vertical dashed lines represent the pretest probabilities of $35 \%$ and $61 \%$ for Hunt and Hess scale grades $1-2$ and $3-5$, respectively. The horizontal dashed lines indicate the posttest probabilities for these $2 \mathrm{Hunt}$ and Hess scale grade classifications. The posttest probabilities for a positive CTP finding remain above the $\mathrm{HHH}$ treatment threshold and do not contribute to treatment decisions. However, the posttest probabilities of a negative CTP finding are below the $\mathrm{HHH}$ treatment threshold and do alter treatment decisions. Thereby, performing CTP to assist in $\mathrm{HHH}$ treatment decisions is considered appropriate in both low and high Hunt and Hess scale grades.

(0.58-0.94) for CTP detecting vasospasm, similar to that in the literature. ${ }^{7,25}$ Construction of the GCPs for Bayesian analysis was based on these test characteristics, suggesting that our results may be applicable to other patient populations with aSAH as well.

Bayesian analysis can assist in treatment decision-making by determining the posttest probability of vasospasm or DCI by using the sensitivity and specificity of CTP along with the pretest probability. The pretest probability is based on all of the patient's clinical and imaging data before CTP. Even though the treatment threshold is variable in different clinical/ disease settings, the principles of its application are the same. At our institution, following a survey of 6 neurologists with expertise in neurointensive care, the median treatment threshold is a posttest probability of $30 \%$ for $\mathrm{HHH}$ and $70 \%$ for intra-arterial therapy. Intra-arterial therapy may be considered as an adjunct to $\mathrm{HHH}$ in patients with posttest probabilities of $\geq 70 \%$. These treatment thresholds have not been empirically validated and are used strictly for illustrative purposes in this study to demonstrate the application and value of Bayesian analysis by using locally derived treatment thresholds. The aim of this study was to indicate the most appropriate range of pretest probabilities for the use of CTP, with the understanding that the range of pretest probabilities in which CTP should be used will vary depending on the individual physician's treatment thresholds.

In this study, a positive CTP finding will increase the posttest probability above the $\mathrm{HHH}$ treatment threshold for patients suspected of DCI with a pretest probability of $\geq 12 \%$ (Fig $1 A$ ). On the other hand, a negative CTP finding will decrease the posttest probability below the treatment threshold in patients with a pretest probability of $<67 \%$. These pretest probabilities indicate the lower and upper boundaries when CTP is most appropriately used in aSAH for detecting DCI. For example, when the pretest probability of DCI is $\geq 67 \%$, a positive or negative CTP finding does not reduce the posttest probability below the HHH treatment threshold and the CTP results will, therefore, not contribute to decisionmaking. Similarly, when the pretest probability of DCI is $<12 \%$, a positive or negative CTP finding does not elevate the posttest probability above the $\mathrm{HHH}$ treatment threshold and thereby does not contribute to decision-making. Similar concepts are also applied to patients suspected of vasospasm (Fig 1B). The results of CTP affect treatment decisions in patients with pretest probabilities of vasospasm between $10 \%$ and $92 \%$, when using the treatment thresholds applied in our study.

In clinical practice, pretest probabilities are informally derived from multiple clinical and imaging predictors before imaging. To date, there are no validated models available for assessing the combined pretest probability by using multiple factors. Therefore, the examples used in this study are focused on pretest probabilities derived from single clinical predictors. Table 2 demonstrates the pretest probabilities for different classification schemes commonly used in clinical practice. The posttest probabilities are calculated for a positive and negative CTP finding. These data can 
be applied to a specific patient for individualization of care. For example, the modified Fisher scale is used in clinical practice to more accurately predict DCI by stratifying patients. ${ }^{26,27}$ In all modified Fisher scale grades, performing CTP contributes to treatment decisions because a positive or negative CTP finding alters the posttest probability above or below the treatment threshold compared with the pretest probability. Similarly, when one uses Hunt and Hess scale grades to determine pretest probability, CTP is also helpful in determining treatment (Fig 2). In this clinical scenario, a negative CTP finding has a greater effect on treatment decisions than a positive CTP finding. However, this is not the case for the Glasgow Coma Scale. Patients with a Glasgow Coma Scale score of $<9$ have a pretest probability that is above the treatment threshold. A positive or negative CTP finding does not alter the posttest probability for treatment decisions. Several clinical classification schemes are presented as the pretest probabilities, given their variable use in clinical practice. Selecting the most appropriate classification scheme for a particular patient population is beyond the scope of this study.

Comparison of the GCPs for DCI (Fig 1A) and vasospasm (Fig $1 B$ ) indicates that CTP is appropriate for a wider range of pretest probabilities in patients suspected of having vasospasm rather than DCI. These data are reflected by the higher positive and negative predictive values of CTP for determining vasospasm. A possible explanation for the differences observed in the test characteristics of CTP may be that the reference standards used to determine DCI and vasospasm were assessed by using primary outcome measures of cerebral infarction and permanent neurologic deficits determined at the end of hospitalization. A CTP, performed typically between days 6 and 8 following aSAH, may have shown a perfusion deficit that subsequently resolved without an infarction or a permanent deficit. On the other hand, a CTP finding may have been negative at days $6-8$, but the patient could have developed ischemia later. Thus, the DCI outcome measures may have worse temporal resolution in relation to the presence or absence of perfusion deficits on CTP. In contrast, for assessment of patients with vasospasm, the CTP and DSA examinations were performed within a short time only in patients with symptoms of vasospasm; that difference may partly explain the superior test characteristics of CTP in determining vasospasm. Another difference noted in the characteristics of the patient group with DSA performed, compared with patients without DSA, is that the DSA group had more patients with neurologic deterioration not explained by other causes because these symptomatic patients were more likely to undergo further testing with DSA and possible intra-arterial treatment.

There are several limitations in this study. The pretest probabilities in the examples were based on single clinical predictors. However, in clinical practice, the pretest probability of a patient represents an estimated likelihood based on the overall evaluation of the patient before CTP, including all available clinical and imaging data. Deriving this overall estimated pretest probability is a complex clinical task because it is affected by a weighted value assigned to each of the clinical and imaging data according to the physician's judgment. Therefore, single clinical predictors were used in this study to illustrate the concepts of applying GCP and to demonstrate the role of CTP in patients with aSAH. Another po- tential limitation is that the results are dependent on the treatment threshold, which may vary among physicians and institutions. However, GCP can be applied in different patient populations by using the specified treatment thresholds established for a specific practice. Furthermore, the sensitivity and specificity of CTP may also vary in different settings, depending on the quality of the imaging and its interpretation. The GCP may also be customized for a local practice setting by using the www. ebr.ie Web site. ${ }^{20}$

\section{CONCLUSIONS}

Given the potential risks associated with over- and underuse of CTP in aSAH for determining vasospasm and DCI, Bayesian analysis can help guide its most appropriate use in this patient population. Our study indicates that over a wide range of pretest probabilities, a positive CTP finding identifies patients who should be carefully considered for $\mathrm{HHH}$ and/or intra-arterial therapy. On the other hand, a negative CTP finding is particularly useful for avoiding treatment-related complications. ${ }^{28,29}$ Treatment thresholds vary in practice and, in lieu of empiric evidence, must be determined by the treating physicians. Future research should be performed to further evaluate these findings in a prospective clinical trial assessing the impact of CTP on patient outcomes.

Disclosures: Holly Delaney—RELATED: Grant: National Institutes of Health, ${ }^{*}$ Comments: National Institutes of Health grant-funded research. Carl E. JohnsonRELATED: Grant: National Institute of Neurological Diseases and Stroke.* Matthew E. Fink—RELATED: Consultancy: Navigant Consultants, Proctor \& Gamble, Maquet; Employment: Editor, Neurology Alert, AHC Media, Expert Testimony: medicolegal, Payment for Development of Educational Presentations: AHC Media-WEBinar. Pina C. Sanelli-RELATED: Grant: National Institute of Neurological Diseases and Stroke.* *Money paid to the institution.

\section{REFERENCES}

1. Vergouwen MD, Vermeulen M, van Gijn J, et al. Definition of delayed cerebral ischemia after aneurysmal subarachnoid hemorrhage as an outcome event in clinical trials and observational studies: proposal of a multidisciplinary research group. Stroke 2010;41:2391-95

2. Schmidt JM, Wartenberg KE, Fernandez A, et al. Frequency and clinical impact of asymptomatic cerebral infarction due to vasospasm after subarachnoid hemorrhage. J Neurosurg 2008;109:1052-59

3. Dankbaar JW, de Rooij NK, Velthuis BK, et al. Diagnosing delayed cerebral ischemia with different $\mathrm{CT}$ modalities in patients with subarachnoid hemorrhage with clinical deterioration. Stroke 2009;40:3493-98

4. Killeen RP, Mushlin AI, Johnson CE, et al. Comparison of CT perfusion and digital subtraction angiography in the evaluation of delayed cerebral ischemia. Acad Radiol 2011;18:1094-100

5. Dankbaar JW, de Rooij NK, Rijsdijk M, et al. Diagnostic threshold values of cerebral perfusion measured with computed tomography for delayed cerebral ischemia after aneurysmal subarachnoid hemorrhage. Stroke 2010;41:1927-32

6. Sanelli PC, Ugorec I, Johnson CE, et al. Using quantitative CT perfusion for evaluation of delayed cerebral ischemia following aneurysmal subarachnoid hemorrhage. AJNR Am J Neuroradiol 2011;32:2047-53

7. Wintermark M, Ko NU, Smith WS, et al. Vasospasm after subarachnoid hemorrhage: utility of perfusion CT and CT angiography on diagnosis and management. AJNR Am J Neuroradiol 2006;27:26-34

8. Aralasmak A, Akyuz M, Ozkaynak C, et al. CT angiography and perfusion imaging in patients with subarachnoid hemorrhage: correlation of vasospasm to perfusion abnormality. Neuroradiology 2009;51:85-93 
9. Cohnen M, Wittsack HJ, Assadi S, et al. Radiation exposure of patients in comprehensive computed tomography of the head in acute stroke. AJNR Am J Neuroradiol 2006;27:1741-45

10. Imanishi Y, Fukui A, Niimi H, et al. Radiation-induced temporary hair loss as a radiation damage only occurring in patients who had the combination of MDCT and DSA. Eur Radiol 2005;15:41-46

11. US Food and Drug Administration. Safety Investigation of CT Brain Perfusion Scans: Update 11/9/2010. www.fda.gov/medicaldevices/ safety/alertsandnotices/ucm185898.htm. Accessed June 1, 2012

12. Maceneaney PM, Malone DE. The meaning of diagnostic test results: a spreadsheet for swift data analysis. Clin Radiol 2000;55: 227-35

13. Joseph L, Reinhold C. Fundamentals of clinical research for radiologists. Introduction to probability theory and sampling distributions. AJR Am J Roentgenol 2003;180:917-23

14. Heffernan EJ, Dodd JD, Malone DE. Cardiac multidetector CT: technical and diagnostic evaluation with evidence-based practice techniques. Radiology 2008;248:366-77

15. Dodd JD. Evidence-based practice in radiology: steps 3 and 4-appraise and apply diagnostic radiology literature. Radiology 2007;242:342-54

16. Sanelli PC, Lev MH, Eastwood JD, et al. The effect of varying userselected input parameters on quantitative values in CT perfusion maps. Acad Radiol 2004;11:1085-92

17. Frontera JA, Fernandez A, Schmidt JM, et al. Defining vasospasm after subarachnoid hemorrhage: what is the most clinically relevant definition? Stroke 2009;40:1963-68

18. Powsner RA, O'Tuama LA, Jabre A, et al. SPECT imaging in cerebral vasospasm following subarachnoid hemorrhage. J $\mathrm{Nucl} \mathrm{Med}$ 1998;39:765-69

19. Lowry R. Clinical calculator 1: from an observed sample-estimates of population prevalence, sensitivity, specificity, predictive values, and likelihood ratios. http://faculty.vassar.edu/lowry/clin1.html. Accessed December 1, 2011

20. Practice Based Learning: Research in to Practice (St. Vincent's University Hospital, Dublin, Ireland). http://www.ebr.ie. Accessed December 1, 2011

21. Yousef K, Crago E, Kuo CW, et al. Predictors of delayed cerebral ischemia after aneurysmal subarachnoid hemorrhage: a cardiac focus. Neurocrit Care 2010;13:366-72

22. Wintermark M, Dillon WP, Smith WS, et al. Visual grading system for vasospasm based on perfusion CT imaging: comparisons with conventional angiography and quantitative perfusion CT. Cerebrovasc Dis 2008;26:163-70

23. Dankbaar JW, Rijsdijk M, van der Schaaf IC, et al. Relationship between vasospasm, cerebral perfusion, and delayed cerebral ischemia after aneurysmal subarachnoid hemorrhage. Neuroradiology 2009;51:813-19

24. Binaghi S, Colleoni ML, Maeder P, et al. CT angiography and perfusion CT in cerebral vasospasm after subarachnoid hemorrhage. AJNR Am J Neuroradiol 2007;28:750-58

25. Wintermark M, Sincic R, Sridhar D, et al. Cerebral perfusion CT: technique and clinical applications. J Neuroradiol 2008;35:253-60

26. Ko SB, Choi HA, Carpenter AM, et al. Quantitative analysis of hemorrhage volume for predicting delayed cerebral ischemia after subarachnoid hemorrhage. Stroke 2011;42:669-74

27. Frontera JA, Claassen J, Schmidt JM, et al. Prediction of symptomatic vasospasm after subarachnoid hemorrhage: the modified Fisher scale. Neurosurgery 2006;59:21-27

28. Warnock NG, Gandhi MR, Bergvall U, et al. Complications of intraarterial digital subtraction angiography in patients investigated for cerebral vascular disease. Br J Radiol 1993;66:855-58

29. Fergusen S, Macdonald RL. Predictors of cerebral infarction in patients with aneurysmal subarachnoid hemorrhage. Neurosurgery 2007;60:658-67 